\title{
Epidemiological Study and Optimal Control for Lumpy Skin Disease (LSD) in Ethiopia
}

\author{
Okey Oseloka Onyejekwe*, Abebe Alemu, Biruk Ambachew, Ayalnesh Tigabie \\ Computational Mechanics and Dynamical Systems Group, Computational Science Program, Addis Ababa University, Arat Kilo \\ Campus, Addis Ababa, Ethiopia \\ Email: ^okuzaks@yahoo.com, abesoftware@gmail.com, hibiruk@yahoo.com, ayutig2008@gmail.com
}

How to cite this paper: Onyejekwe, O.O., Alemu, A., Ambachew, B. and Tigabie, A. (2019) Epidemiological Study and Optimal Control for Lumpy Skin Disease (LSD) in Ethiopia. Advances in Infectious Diseases, 9, 8-24.

https://doi.org/10.4236/aid.2019.91002

Received: December 28, 2018

Accepted: February 18, 2019

Published: February 21, 2019

Copyright $\odot 2019$ by author(s) and Scientific Research Publishing Inc. This work is licensed under the Creative Commons Attribution International License (CC BY 4.0).

http://creativecommons.org/licenses/by/4.0/

\begin{abstract}
Lumpy skin disease (LSD) is an infectious, fatal skin disease of cattle caused by a virus of the family Poxviridae (genus Capripox). In addition, severely affected animals suffer from reduced weight, cessation of milk production and infertility. The aim of this paper is to computationally apply epidemiological (SEIR) and optimal control (OC) techniques to study the transmission and the impact of vaccination on LSD. Based on our numerical experiments, we were able to deduce the overall impact of the optimal strategy adopted for this study on the cattle population for vaccination rates within the range of $0 \leq v \leq 0.85$. It is shown that the vaccination as a control strategy significantly reduced the effects of LSD on the cattle population if properly managed and that an optimal performance of the control strategy adopted hererin is achieved at an approximate value of $v=0.6$.
\end{abstract}

\section{Keywords}

Lumpy Skin Disease, LSD, Optimal Control, Epidemiology, SEIR

\section{Introduction}

LSD has an economical importance because of permanent damage to hides. It has been reported ([1] [2]) that $10 \%$ of animals exposed to LSD experience abortion. According to [3], lumpy skin disease (LSD) is an exhaustive viral disease and a pox disease of cattle. It is characterized by fever, nodules on the skin, mucous membranes in internal organs, enlarged lymph nodes and odema of the skin ([3] [4]). Due to chronic debility in affected animals high economic losses occur. The clinical syndrome of LSD was first described in Zambia in 1929. More cases also occurred between 1943 and 1945 in Botswana, Zimbabwe and the republic of South Africa ([1] [3] [5] [6]). Transmission of LSD is by insect 
vectors (through biting flies) found among cattle sharing similar grazing and watering areas. And those that congregate in the same barn may be contaminated by the viruses in saliva of the infected animals or ingestion of already contaminated food or by teratogenic agents and suckling calves ([7] [8]). The incidence of LSD occurrence is high during wet seasons [3].

LSD was first observed in Ethiopia in the northwestern part of the country (southwest of Lake Tana) in 1983, [1]. It occurs in all agro-climatic conditions and has the potential to extend its boundaries. Good understanding of epidemiology, economic significance and control mechanisms of the disease will enhance suitable control measures. The disease could be diagnosed using appropriate serological and molecular techniques. Effective control measures can be achieved through mass vaccination, separation and culling of infected animals, ([9] [10] [11]). It is therefore important to formulate an LSD model with optimal control policy to eradicate LSD epidemic and minimize the cost associated for vaccination strategy [3].

Most studies of epidemic control of LSD focus on increasing immunization coverage (vaccination coverage) in a population to control the disease, but they do not consider how this parameter affects the strategy over a period of time. Though some of these studies have considered vaccination strategy at different levels of immunization, none of them has studied optimal vaccination strategy for LSD disease in Ethiopia. Hence the general objective of this study aims at addressing these shortfalls in order to make the number of infected animals as small as possible.

\section{Epidemiological Model Formulation}

\section{SEIR Model and Equation Formulation}

All Epidemiological ODE models of disease transmission are continuous time compartment level models [12]. The model considered herein is compartmentalized into:

S: susceptible portion of population,

E: exposed population.

I: the infected population.

$\mathrm{R}$ : the recovered population.

Newly born population merge into the $\mathrm{S}$ compartment at the rate of alpha $(\alpha)$, which represents the birth rate. The infected population are those individuals who can contract the disease. However for the SEIR model, we also consider the exposed population that is those individuals who have the disease but are not yet infectious. After treatment the infected population will move into R-Compartment, at the rate of omega $\omega$ i.e. the recovery rate. Cattles can lose their immunity after some time. Let us assume a rate of $\theta$. As a result, that population moves from $\mathrm{R}$ into the $\mathrm{S}$ compartment. Hence the following S-E-I-R-S Epidemiological model without control is shown in Figure 1 as:

The system is represented by the temporal vectors $S(t), E(t), I(t), R(t)$; and is 
characterized by the following assumptions

- The population is homogeneous.

- No demographics,(immigration and emigration) consideration

- Birth rate and natural death rate are constant. Mathematically this means

$$
S(t)+E(t)+I(t)+R(t)=N
$$

where $N$ is the total population. This facilitates the expression of one of the variables in terms of the others and the conserved constant $N$. Table 1 shows the description of the model parameters and variables applied in this study.

$$
\begin{gathered}
\frac{\mathrm{d} S}{\mathrm{~d} t}=\alpha N-\beta S I+\theta R-\phi S \\
\frac{\mathrm{d} E}{\mathrm{~d} t}=\beta S I-\gamma E-\phi E \\
\frac{\mathrm{d} I}{\mathrm{~d} t}=\gamma E-\omega I-\phi I
\end{gathered}
$$

\begin{tabular}{|c|c|c|}
\hline No. & parameter & Description \\
\hline 1 & $N$ & Total population \\
\hline 2 & $S$ & Susceptible to be expose for infection those of who interact with the disease \\
\hline 3 & $E$ & Exposed population that are infected but have not yet become infectious \\
\hline 4 & $I$ & $\begin{array}{l}\text { Infected by the disease who are capable of transmitting the disease to any } \\
\text { susceptible individuals. }\end{array}$ \\
\hline 5 & $R$ & $\begin{array}{l}\text { Individuals recovered from infection } \\
\text { who are temporarily immune from the infection }\end{array}$ \\
\hline 6 & $\alpha$ & Birth rate from the total population $N$ \\
\hline 7 & $\beta$ & The rate of population exposed to the disease. \\
\hline 8 & $\gamma$ & The rate which will be infected from the population of exposed \\
\hline 9 & $\omega$ & $\begin{array}{l}\text { The rate of getting immunity after sort of treatment } \\
\text { in move to the recovered compartment from infected compartment. }\end{array}$ \\
\hline 10 & $\theta$ & $\begin{array}{l}\text { The rate of populations loss their immunity and moved to the susceptible } \\
\text { compartment. }\end{array}$ \\
\hline 11 & $\phi$ & The rate of natural death \\
\hline
\end{tabular}

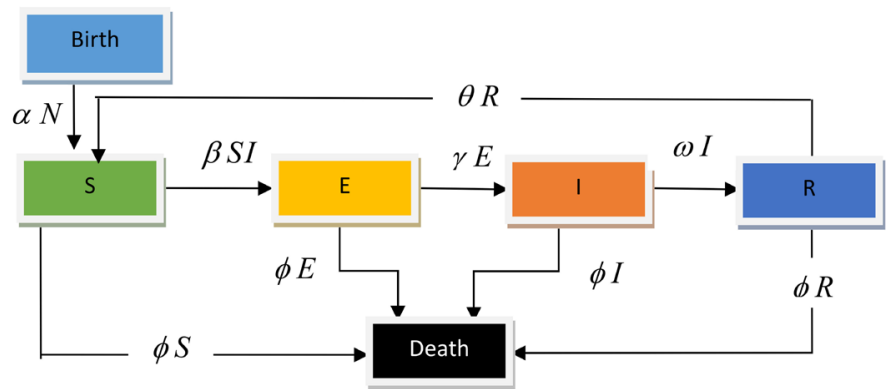

Figure 1. SEIRS Model without Control.

Table 1. Descriptions for variables for the model. 


$$
\frac{\mathrm{d} R}{\mathrm{~d} t}=\omega I-\theta R-\phi R
$$

Note that the natural death rate on each of the compartment for this paper is the same, which is

$$
\phi S+\phi E+\phi I+\phi R=\phi(S+E+I+R)=\phi N
$$

\section{Optimal Control}

Optimal control deals with the problem of finding a control law for a given system such that a certain optimal criterion is achieved. A control problem includes a function of state and control variable. An optimal control is a set of differential equations describing the paths of the control variables that minimize the cost function. The control can be derived using Pontryagins maximum principles (a necessary condition also known as Pontryagins minimum principle or simply Pontryagins Principle), or by solving the Hamilton-Jacobi-Bellamn Equations (a sufficient condition), [13]. This boundary-value problem actually has a special structure because it arises from taking the derivative of a Hamiltonian. Thus, the resulting dynamical system is a Hamiltonian system of the form

$$
\begin{gathered}
\dot{x}=\frac{\partial H}{\partial \lambda} \\
\dot{\lambda}=-\frac{\partial H}{\partial x}
\end{gathered}
$$

where

$$
H=L+\lambda^{\mathrm{T}} a-\mu^{\mathrm{T}} b
$$

is an augmented Hamiltonian [14]. For this study, vaccination is applied as an optimal control and is denoted as $u$.

\section{Numerical Solutions of Optimal Control Problems}

Consider the optimal control problem

$$
\begin{gathered}
\max U \int_{t_{0}}^{t_{1}} f(t, x(t), u(t)) \mathrm{d} t \\
\text { subject to : } \quad x^{\prime}=g(t, x(t), u(t)) \\
x\left(t_{0}\right)=x_{0}, x\left(t_{1}\right) \quad \text { free }
\end{gathered}
$$

Any solution to the above optimal control problem must also satisfy

$$
\begin{gathered}
x^{\prime}(t)=g(t, x(t), u(t)), x\left(t_{0}\right)=x_{0} \\
\lambda(t)=-\frac{\delta H}{\delta x}=-\left(f_{x}(t, x, u)+\lambda(t) g_{x}(t, x, u)\right), \lambda\left(t_{1}\right)=0 \\
0=\frac{\delta H}{\delta x}=-\left(f(t, x, u)+\lambda(t) g_{u}(t, x, u)\right), \text { at } u^{*}
\end{gathered}
$$

Equation (6), the optimal condition, can usually be manipulated to find a re- 
presentation of $u^{*}$ in terms of $t, x$, and $\lambda$.

An optimal control with bounded control can be written in the form

$$
\begin{aligned}
& \max _{u} J(x(t),(u))=\int_{t_{0}}^{t_{f}} f(t, x(t), u(t)) \mathrm{d} t \\
& \text { Subject to }\left\{\begin{array}{l}
x^{\prime}=g(t, x(t), u(t)) \\
x\left(t_{0}\right)<x_{0} \\
a \leq u(t) \leq b
\end{array}\right.
\end{aligned}
$$

where $a, b$ are fixed real constants and $a<b$.

Proposition (Necessary conditions):- If $u^{*}(t)$ and $x^{*}(t)$ are optimal for Equations $(16,17)$, then there exists a piecewise differentiable adjoint variable $\lambda(t)$ such that

$$
H\left(t, x^{*}(t), u(t), \lambda(t)\right) \leq H\left(t, x(t), u^{*}(t), \lambda(t)\right)
$$

For all controls $\mathrm{u}$ at each time $t$, where $H$ is the Hamiltonian, [13].

$$
\begin{gathered}
\lambda^{\prime}(t)=-\frac{\delta H\left(x^{*}(t), u^{*}(t), \lambda(t)\right)}{\delta x} \text { (adjoint condition) } \\
\lambda\left(f_{t}\right)=0 \text { (transversality condition) }
\end{gathered}
$$

By an adaptation of the Pontryagin's Maximum Principle (PMP), the OC must satisfy (optimal condition):

$$
u^{*}=\left\{\begin{array}{l}
a \text { if } \frac{\delta H}{\delta u}<0, \\
a<\frac{\delta H}{\delta u}<b, \text { if } \frac{\delta H}{\delta u}=0, \\
b<\frac{\delta H}{\delta u}<a, \text { if } \frac{\delta H}{\delta u}>0,
\end{array}\right.
$$

If we have a minimization problem, then $u^{*}$ is chosen to minimize $H$ point wise.

If $\frac{\partial^{2} H}{\partial u^{2}}<0$ at $u^{*}$ then $u$ is the maximization of the problem, while $\frac{\partial^{2} H}{\partial u^{2}}<0$ goes with minimization.

The optimal control $\frac{\delta H}{\delta u}=0$ obtained without truncation, and is bounded by $a$ and $b$ such that:

$$
u^{*}(t)=\min \left\{a, \max \left\{b, \frac{\delta H}{\delta u}\right\}\right\}
$$

Runge-Kutta (RK4) routine is applied for the numerical solution. It is required that:

$\sum_{i=1}^{n}\left|u(i)-u_{\text {old }}(i)\right|$ be small, in addition to guaranteeing that the relative error between the current and the previous computed scalars be negligible, i.e. 


$$
\frac{\left|u-u_{\text {old }}\right|}{|u|} \leq \delta
$$

where $\delta$ is the accepted tolerance? The optimal control problem can now be written in a standard form:

$$
\begin{array}{r}
\max \int_{0}^{1} A x(t)-B u^{2}(t) \mathrm{d} t \\
\text { Subject to:- } x^{\prime}(t)=-\frac{1}{2} x^{2}(t)+C u(t) \\
x(0)=x_{0} \text { fixed } x(1) \text { free }
\end{array}
$$

In order for this to be a maximization problem, we require $B>0$. The Hamiltonian given by

$$
H=A x-B u^{2}-\frac{1}{2}+C \lambda u
$$

By applying the optimal condition

$$
\begin{gathered}
0=\frac{\delta H}{\delta u}=-2 B u+C \lambda \Rightarrow u^{*}=\frac{C \lambda}{2 B} \\
x^{\prime}(t)=-\frac{1}{2} x^{2}+C u, x(0)=x_{0} \\
\lambda^{\prime}(t)=-A+x \lambda, \lambda(1)=0
\end{gathered}
$$

The Runge-Kutta forward sweep yields the transient profile of the dependent variable. The new $u^{*}$ is calculated by setting

$$
\begin{gathered}
u_{1}=C * \frac{\lambda}{2 * B} \\
u=0.5 *\left(u_{1}+u_{\text {old }}\right)
\end{gathered}
$$

\section{ODE Formulation for Optimal Control}

\subsection{Modification of SEIRS Model}

A typical optimal control problem requires a performance index or cost function $(J[x(t), u(t)])$, a set of state variables $(x(t) \in X)$ and a set of control variables $(u(t) \in U)$ in a time $t, t_{0} \leq t \leq t_{f}$. A major goal of the work reported herein, is to find a piecewise continuous control $u(t)$ (vaccination) and the associated state variable $x(t)$ to minimize a given objective functional. This strategy will directly impact on the susceptible " $S$ " and the recovered " $R$ " compartments in the overall mathematical formulation as shown below.

$$
\begin{gathered}
\frac{\mathrm{d} S}{\mathrm{~d} t}=\alpha\left(1-v_{1}\right) N-\beta S I-v_{2} S+\theta R-\phi S \\
\frac{\mathrm{d} E}{\mathrm{~d} t}=\beta S I-\gamma E-\phi E \\
\frac{\mathrm{d} I}{\mathrm{~d} t}=\gamma E-\omega I-\phi I
\end{gathered}
$$




$$
\frac{\mathrm{d} R}{\mathrm{~d} t}=\alpha v_{1} N+v_{2} S+\omega I-\theta R-\phi R
$$

In the above equations, vaccination is applied during $\mathrm{g}$ birth $v_{1}$, and for populations in the S-compartment $v_{2}$, thus vaccinated populations moved to recovered compartment as $\alpha v_{1} N$ and $v_{2} S$. However, for this paper, we applied vaccination on $\mathrm{S}$-compartment only as $(v S)$, and the formulation is given as below.

$$
\begin{gathered}
\frac{\mathrm{d} S}{\mathrm{~d} t}=\alpha N-\beta S I-v S+\theta R-\phi S \\
\frac{\mathrm{d} E}{\mathrm{~d} t}=\beta S I-\gamma E-\phi E \\
\frac{\mathrm{d} I}{\mathrm{~d} t}=\gamma E-\omega I-\phi I \\
\frac{\mathrm{d} R}{\mathrm{~d} t}=v S+\omega I-\theta R-\phi R
\end{gathered}
$$

With initial conditions,

$$
\begin{aligned}
& S(0)=S_{0} \geq 0, E(0)=E_{0} \geq 0 \\
& I(0)=I_{0} \geq 0, R(0)=R_{0} \geq 0
\end{aligned}
$$

The above equations are illustrated graphically in Figure 2 as:

The optimal problem (which is the main objectives of this paper) is to minimize the objective (cost) functional ( $/$ ) by considering the costs of vaccination for susceptible population given by:

$$
J(u)=\int_{0}^{t}\left(A I+\frac{1}{2} B u^{2}\right) \mathrm{d} t
$$

where:-

$t$ : represents the vaccination period

$A$ : a parameter that balancing cost factors due to the size of ineffective

$B$ : represents the weight attached on the cost of vaccination

$A$ and $B$ can also be weight parameters describing the comparative importance of the two terms (disease burden and cost) in the functional respectively.

A high value of $A$ means that it is more important to reduce the disease burden than to reduce the vaccination costs. A less value of $B$ means that it is more important to reduce the vaccination costs than to reduce the disease burden. The control $u$ is the percentage of the susceptible that is vaccinated per unit time. Thus, $u$ lies between 0 and 1 while $u_{\max }$ will depend on the amount of resources available to implement each of the control measures.

If $u=0$, then no vaccination is done which is uncontrolled. If $u=1$, then all susceptible population is vaccinated.

The rate of vaccination is assumed to take values in $[0,0.9]$ instead of $[0,1]$ to eliminate the case where the entire susceptible population is vaccinated.

The vaccination cost could include the cost of the vaccine, cost of syringes, cost of safety boxes, the vaccine storage cost, and other related overheads. 


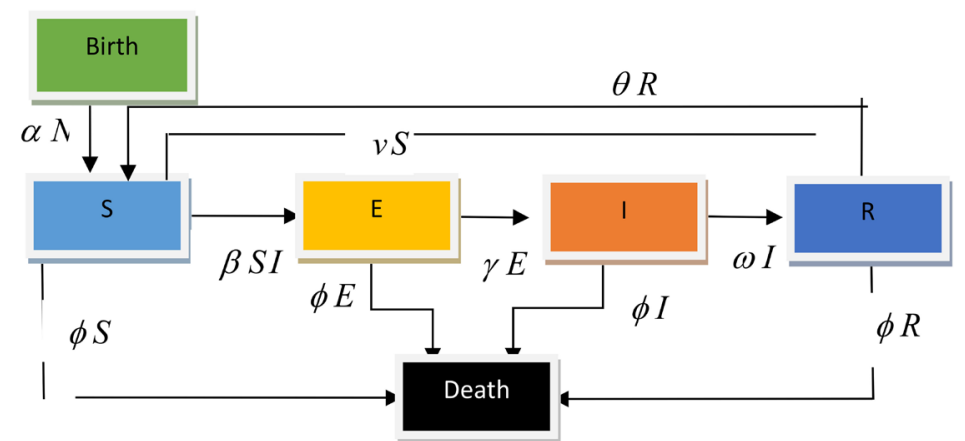

Figure 2. Optimal control with vaccination.

Hence, the Optimal Control problem of the nonlinear dynamics of SEIRS LSD epidemic model studied herein is given by;

$$
\min _{u} J(u)=\min \int_{0}^{t}\left(A I+\frac{1}{2} B u^{2}\right) \mathrm{d} t
$$

where $U=u(t): 0 \leq u \leq u_{\max }<1, t \in[0, t]$.

For the Hamiltonian function:

$$
\begin{aligned}
& H\left(t, S, E, I, R, \lambda_{1}, \lambda_{2}, \lambda_{3}, \lambda_{4}\right) \\
& =f(t, S, E, I, R, u, v)+\sum_{i=1}^{4} \lambda_{i} g_{i}(t, S, E, I, R, u, v) \\
& =f(t, S, E, I, R, u, v)+\lambda_{1} \frac{\mathrm{d} S}{\mathrm{~d} t}+\lambda_{2} \frac{\mathrm{d} E}{\mathrm{~d} t}+\lambda_{3} \frac{\mathrm{d} I}{\mathrm{~d} t}+\lambda_{4} \frac{\mathrm{d} R}{\mathrm{~d} t}
\end{aligned}
$$

Then by substituting each of the derivatives in Equation (41) into Equation (42) and by applying PMP to the Hamiltonian, we have:

$$
\begin{aligned}
H= & \left(A I+\frac{1}{2} B u^{2}\right)+\lambda_{1}(\alpha N-\beta S I-u S+\theta R-\phi S) \\
& +\lambda_{2}(\beta S I-\gamma E-\phi E)+\lambda_{3}(\gamma E-\omega I-\phi I) \\
& +\lambda_{4}(u S+\omega I-\theta R-\phi R)
\end{aligned}
$$

The Adjoint functions with respect to the dependent variables are:

For $S$ :

$$
\begin{aligned}
& \lambda_{1}^{*}=-\frac{\delta H}{\delta S} \\
& \Rightarrow \lambda_{1}^{*}=-\left[\lambda_{1}(\beta I-u-\phi)+\lambda_{2} \beta I+\lambda_{4} u\right] \\
& \Rightarrow \lambda_{1}^{*}=\lambda_{1}(-\beta I+u+\phi)-\lambda_{2} \beta I-\lambda_{4} u
\end{aligned}
$$

Adjoint function with respect to $E$

$$
\begin{aligned}
& \lambda_{2}^{*}=-\frac{\delta H}{\delta E} \\
& \Rightarrow \lambda_{2}^{*}=-\left[\lambda_{2}(-\gamma-\phi)+\lambda_{3} \gamma\right] \\
& \Rightarrow \lambda_{2}^{*}=\lambda_{2}(\gamma+\phi)-\lambda_{3} \gamma
\end{aligned}
$$

Adjoint function with respect to $I$ 


$$
\begin{aligned}
& \lambda_{3}^{*}=-\frac{\delta H}{\delta I} \\
& \Rightarrow \lambda_{3}^{*}=-\left[A+\lambda_{1}(-\beta S)+\lambda_{2} \beta S+\lambda_{3}(-\phi-\omega)+\lambda_{4} \omega\right] \\
& \Rightarrow \lambda_{3}^{*}=-A+\lambda_{1} \beta S-\lambda_{2} \beta S+\lambda_{3}(\phi+\omega)-\lambda_{4} \omega
\end{aligned}
$$

Adjoint function with respect to $R$

$$
\begin{aligned}
& \lambda_{4}^{*}=-\frac{\delta H}{\delta R} \\
& \Rightarrow \lambda_{4}^{*}=-\left[\lambda_{1}(\theta)+\lambda_{4}(-\theta-\phi)\right] \\
& \Rightarrow \lambda_{4}^{*}=-\lambda_{1} \theta+\lambda_{4}(\theta+\phi)
\end{aligned}
$$

From the Equation (43 to 47); we obtain:

$$
\begin{aligned}
& \lambda_{1}^{*}=\lambda_{1}(-\beta I+u+\phi)-\lambda_{2} \beta I-\lambda_{4} u \\
& \lambda_{2}^{*}=\lambda_{2}(\gamma+\phi)-\lambda_{3} \gamma \\
& \lambda_{3}^{*}=-A+\lambda_{1} \beta S-\lambda_{2} \beta S+\lambda_{3}(\phi+\omega)-\lambda_{4} \omega \\
& \lambda_{4}^{*}=-\lambda_{1} \theta+\lambda_{4}(\theta+\phi)
\end{aligned}
$$

With transversality conditions we have $\lambda_{i}(T)=0, i=1,2,3,4$

Next, we consider an optimal condition for determining the characterization of the optimal control $u^{*}$. The optimal condition by differentiating the Hamiltonian $H$ with respect to $u$ based on the Equation (27) for each control $u^{*}$. We have the following optimal system:

$$
\frac{\delta H}{\delta u}=0 \text { at } u=u^{*} \Rightarrow B u+\lambda_{1}(-S) \text {, at } u=u^{*}
$$

then we have $u^{*}(t)=\lambda_{1} \frac{S}{B}$ taking into account the bound $u^{*}$ and its characterization obtain;

$$
u^{*}= \begin{cases}0 & \text { if } \frac{\delta H}{\delta u}<0, \\ \lambda_{1} \frac{S}{B} & \text { if } \frac{\delta H}{\delta u}=0 \\ 0.9 & \text { if } \frac{\delta H}{\delta u}>0\end{cases}
$$

the optimal control $u^{*}$ can now be put in a compact form:

$$
u^{*}=\max \left(\min \left(\lambda_{1} \frac{S}{B}, 0.9\right), 0\right)
$$

Hence using the characterization of the optimal control, we now have following optimal system

$$
\begin{gathered}
S^{\prime}=\alpha N-\beta S I-u S+\theta R-\phi S \\
E^{\prime}=\beta S I-\gamma E-\phi E \\
I^{\prime}=\gamma E-\omega I-\phi I \\
R^{\prime}=u S+\omega I-\theta R-\phi R \\
S(0)=S_{0} \geq 0, E(0)=E_{0} \geq 0, I(0)=I_{0} \geq 0, R(0)=R_{0} \geq 0
\end{gathered}
$$


To determine the optimal vaccination, we use a forward-backward sweep numerical method. The model solves the SEIRS Equations (36 to 39) using fourth order Runge-Kutta (RK4) method. The values of all the accompanying parameters necessary for the successful numerical solution of the governing equations are given in Table 2.

\subsection{Numerical Results and Discussion}

In Figure 3, no optimal control was applied. The number of populations in $S$ is dramatically decreased. Whereas the number of Exposed populations exhibits an increase with time $(t)$. After some time, the population shows an increase in E-compartment. As time progresses, the number of population in I compartment increases, while the number of population in R-decrease. This suggests an endemic situation.

Unlike the previous case, Figure 4 displays the effect of optimal control (vaccination) on the susceptible population. As a consequence of vaccination, there is a movement of the vaccinated population from the $S$ compartment to the recovery compartment. This is confirmed by an increase in the recovery population in the graph. We hasten to comment that irrespective of any control strategy, the population in the $\mathrm{S}$ compartment displays a decline as time progresses, but the rate of decrease when vaccination is applied is slower. With the population in the S-compartment vaccinated, recovery is enhanced. This shows that LSD disease can be controlled with vaccination. In addition, Figure 4 displays the response of the population in the S-compartment, for optimal control and non optimal control applications. For both cases, the size of the population decreases at different rates. The profiles for both cases clearly demonstrate the effect of the application of this treatment strategy for the susceptible population.

Table 2. Assigned values for model parameters.

\begin{tabular}{cccc}
\hline Symbols & Description & Value & Reference \\
\hline$\beta$ & Exposed rate & 0.02 & {$[2]$} \\
$\gamma$ & Infection rate & 0.29 & {$[3]$} \\
$\omega$ & Recovered rate & 0.3 & Estimation \\
$\phi$ & Natural death rate & 0.00002 & {$[6]$} \\
$\theta$ & Rate of losing immunity & 0.00002 & Estimation \\
$\alpha$ & and move to S from R Compartment. & & {$[6]$} \\
$A$ & Birth rate & 0.0004 & Estimation \\
$B$ & Balancing cost factor & 1000 & Estimation \\
{$[0, t]$} & The weight of the cost of vaccination & 200 & {$[6]$} \\
{$[0, u]$} & Vaccination period & {$[0,12]$ months } & {$[6]$} \\
$S_{0}$ & Vaccination rate & {$[0,0.9]$} & {$[3]$} \\
$E_{0}=I_{0}=R_{0}$ & Initial Susceptible population & 3000 & {$[11]$} \\
\hline
\end{tabular}




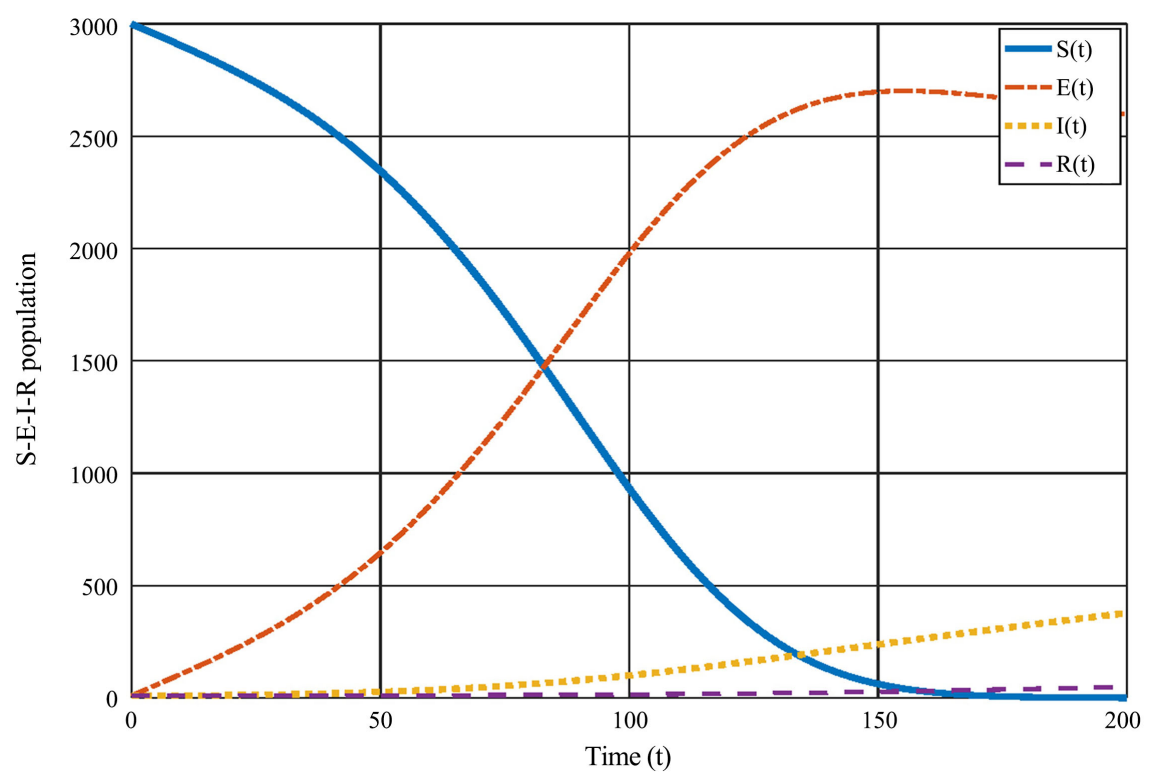

Figure 3. SEIRS without Optimal control.

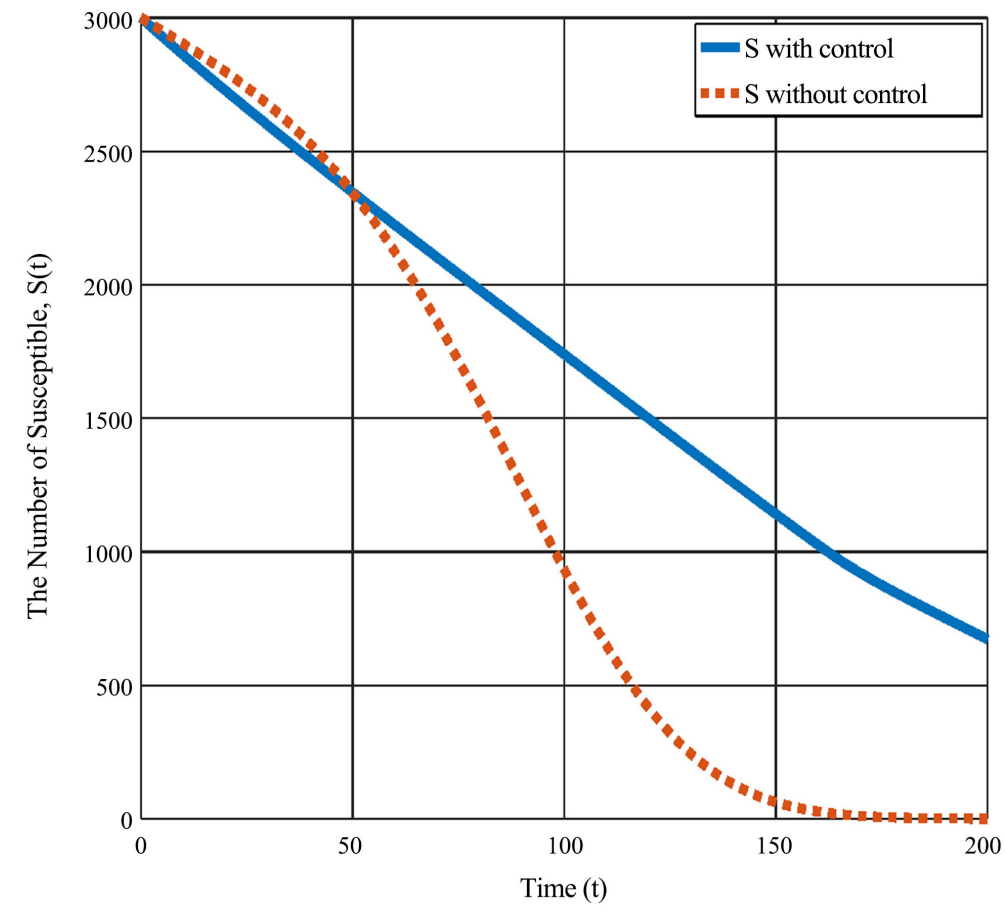

Figure 4. S-compartment with optimal control and without optimal control.

Figure 5 shows the effect of vaccination as a control measure on the infected population. The sharp decline in the infected cattle population demonstrates that if vaccination is properly applied it sure will turn out as a utilitarian strategy to control LSD spread in cattle, The same can also be said of the "E" or the exposed population (see Figure 6). There is less population in the exposed compartment as a result of the application of vaccination. Both cases demonstrate the beneficial effects of vaccination with time. 


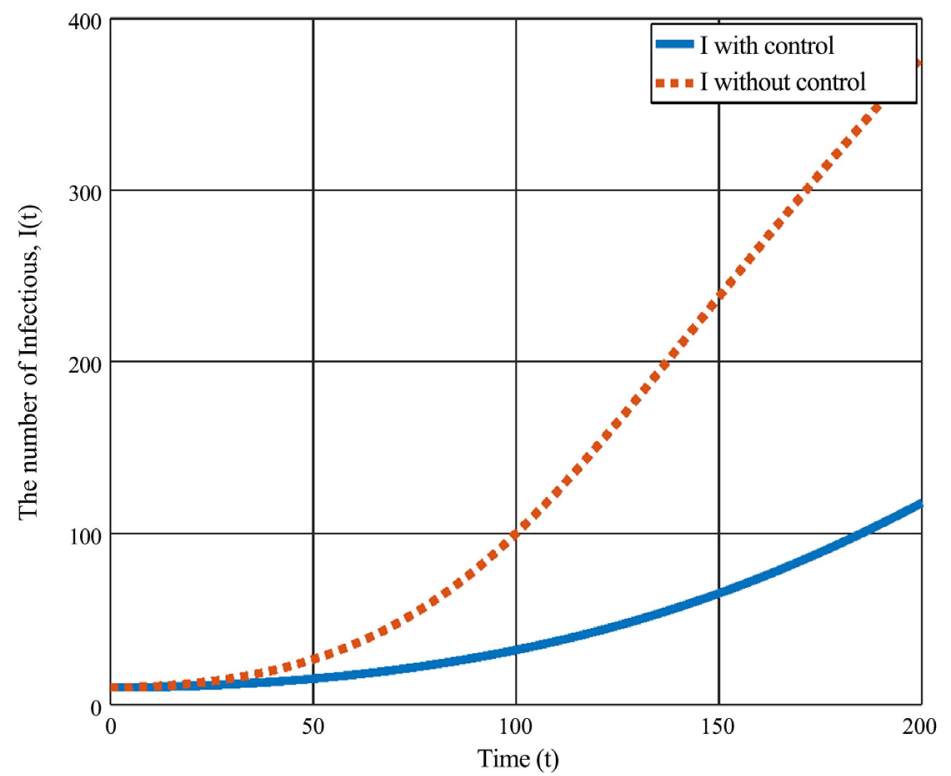

Figure 5. I-compartment with optimal control and without optimal control.

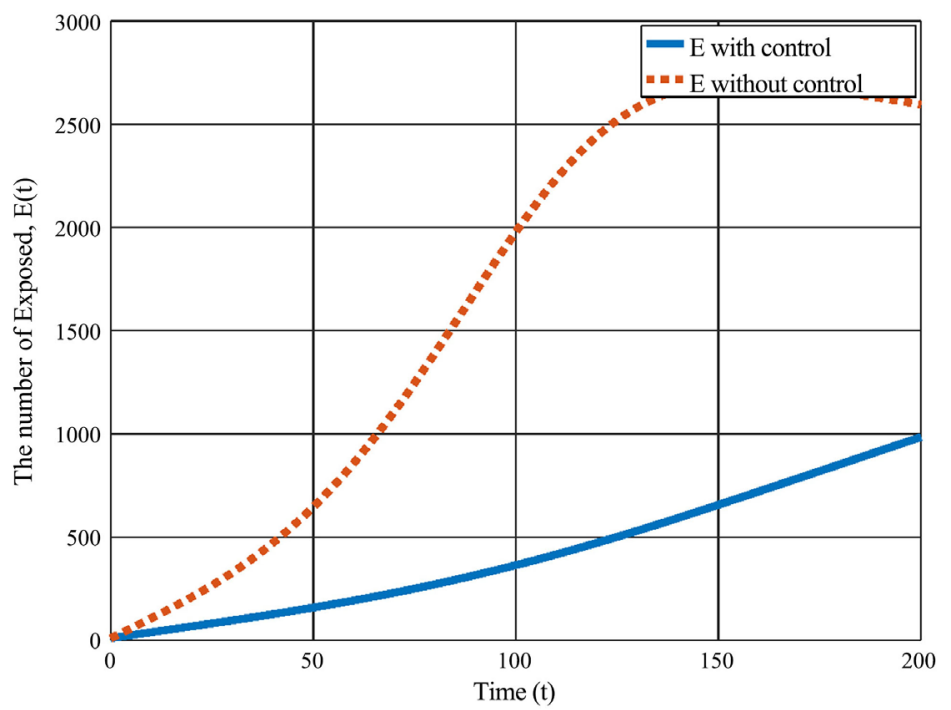

Figure 6. E-compartment with optimal control and without optimal control.

Figure 7 shows a dramatic rise in the " $R$ " population resulting from the application of vaccination. This reflects an inflow from virtually all the compartments of the model into the " $R$ " population when control is applied. This again shows that vaccination as intervention strategy has a great impact on controlling LSD among cattle.

For demonstration purposes, we present the effects of different vaccination rates $(0 \leq v \leq 0.85)$ on the SEIR profiles in order to assess the impact on the overall control strategy adopted for this study.

Figures 8-13 show a continuous decrease in the " $\mathrm{S}$ " population for increasing vaccination rates. This is solely because vaccination plays a big role in decreasing the susceptible population for any successful control strategy. As the susceptible 
population decreases, they move to both the recovered (" $R$ " population) and the exposed (the "E" i.e. the exposed or the latent population). There is a gradual rise in the "E" population as indicated in Figure 8. Initially it exhibits a higher profile than the "R" population (see Figure 9 and Figure 10). Figures 11-13 show that it finally catches up with and surpasses the exposed population. The actual point of transition happens around $v=0.6$. Given the parameters adopted in this study this can be considered as an optimal point of application and the decision as to whether to continue increasing the vaccination rate will depend on other consideration among which will be that availability of funds. From Figures 8-13, it can also be observed that the profile of the infected population (the "I" compartment) remains the lowest when compared to the dynamic profiles exhibited by other compartments.

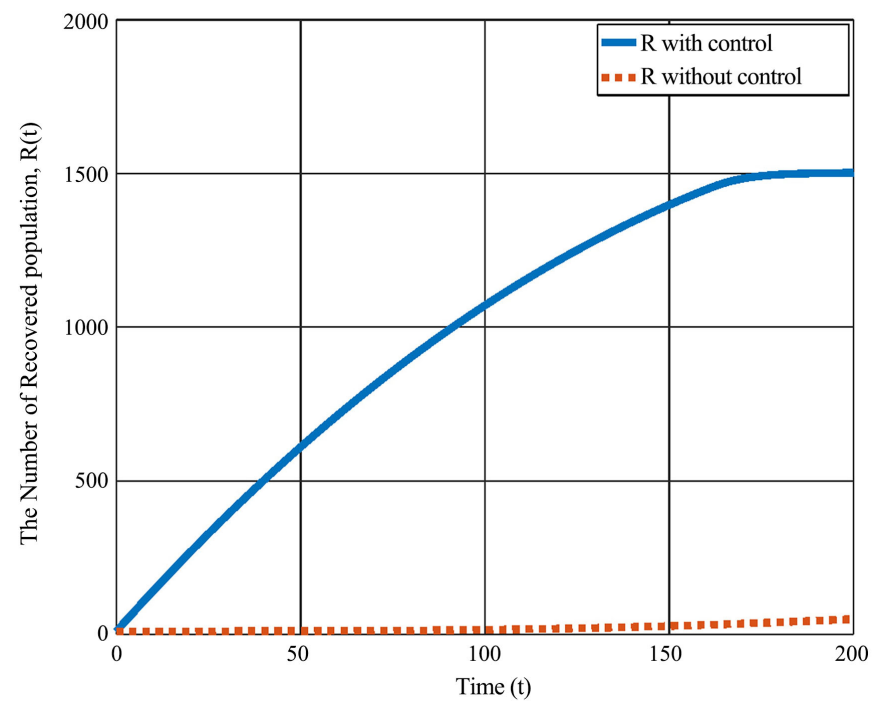

Figure 7. R-compartment with OC and without OC.

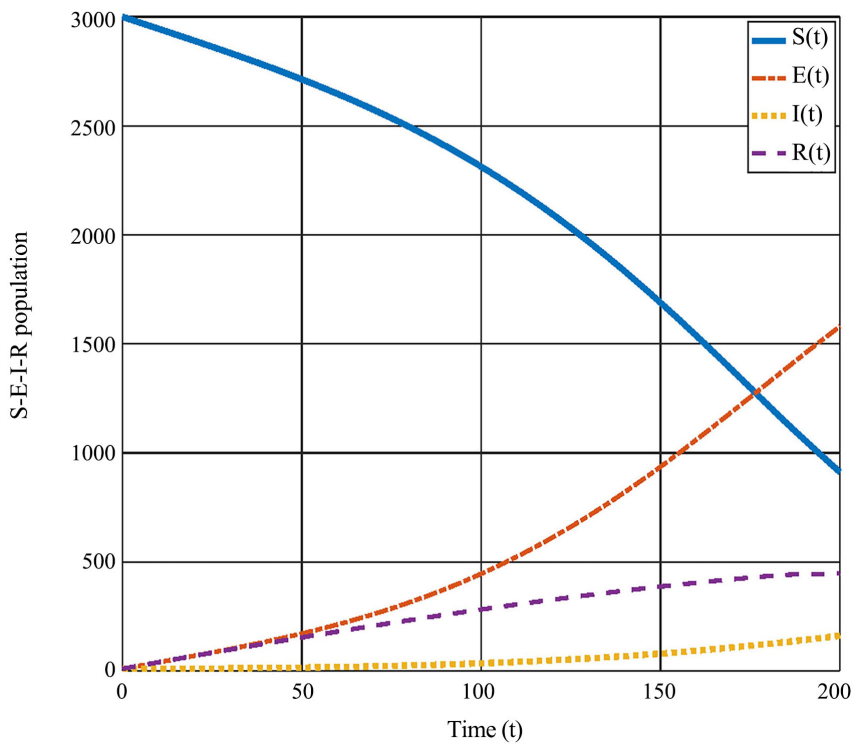

Figure 8. SEIR-compartment with optimal control at $u[0,0.2]$. 


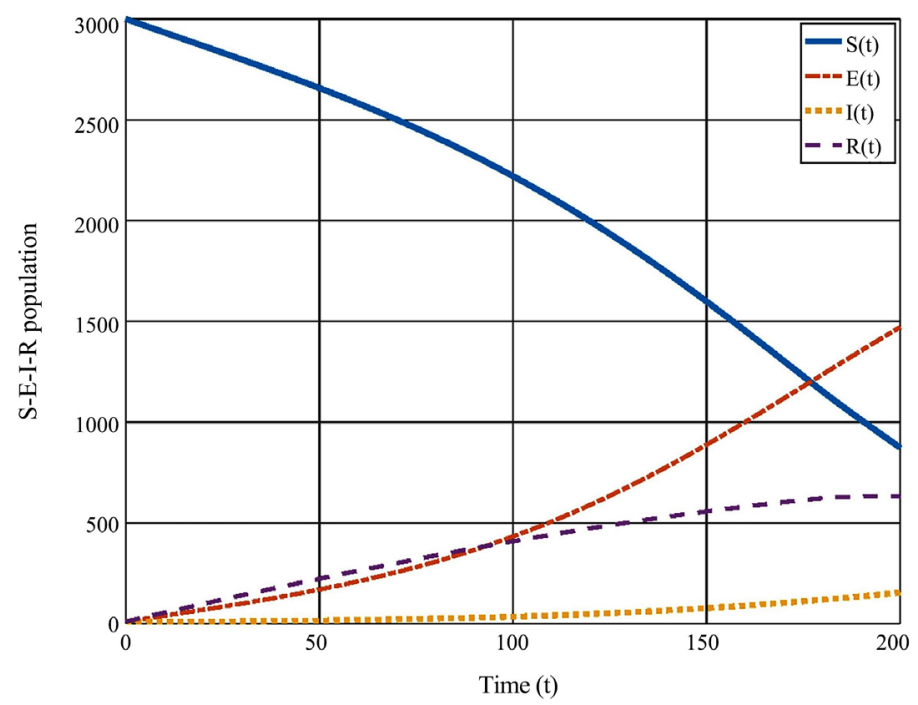

Figure 9. SEIR-compartment with optimal control at $u[0,0.3]$.

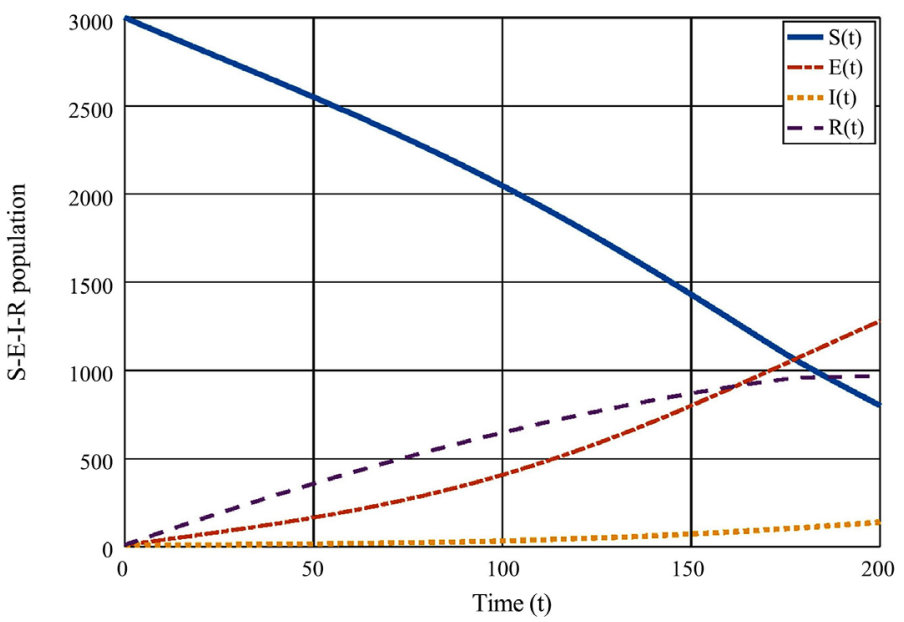

Figure 10. SEIR-compartment with optimal control at $u[0,0.5]$.

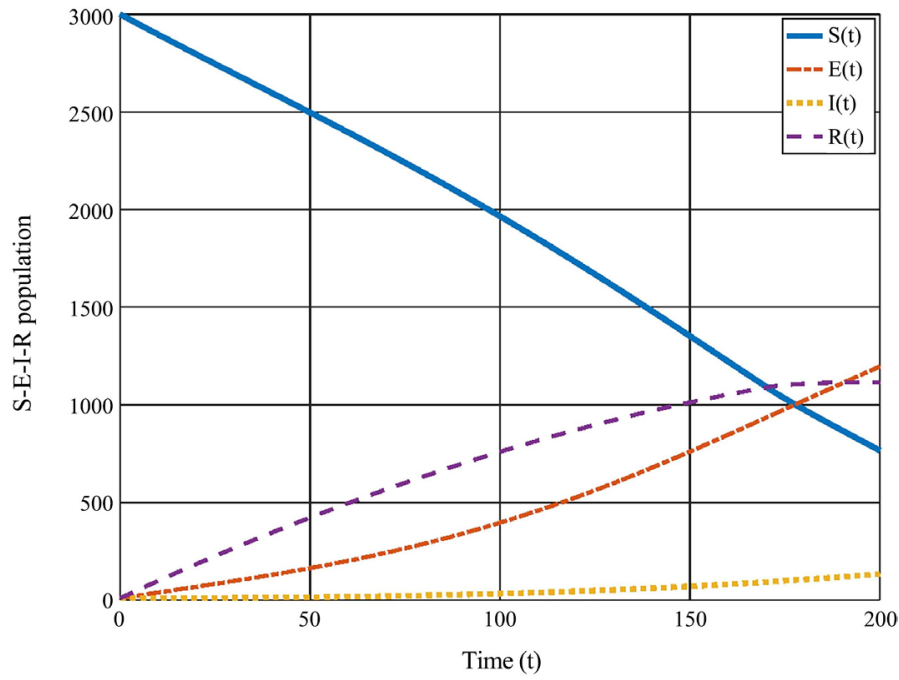

Figure 11. SEIR-compartment with optimal control at $u[0,0.6]$. 


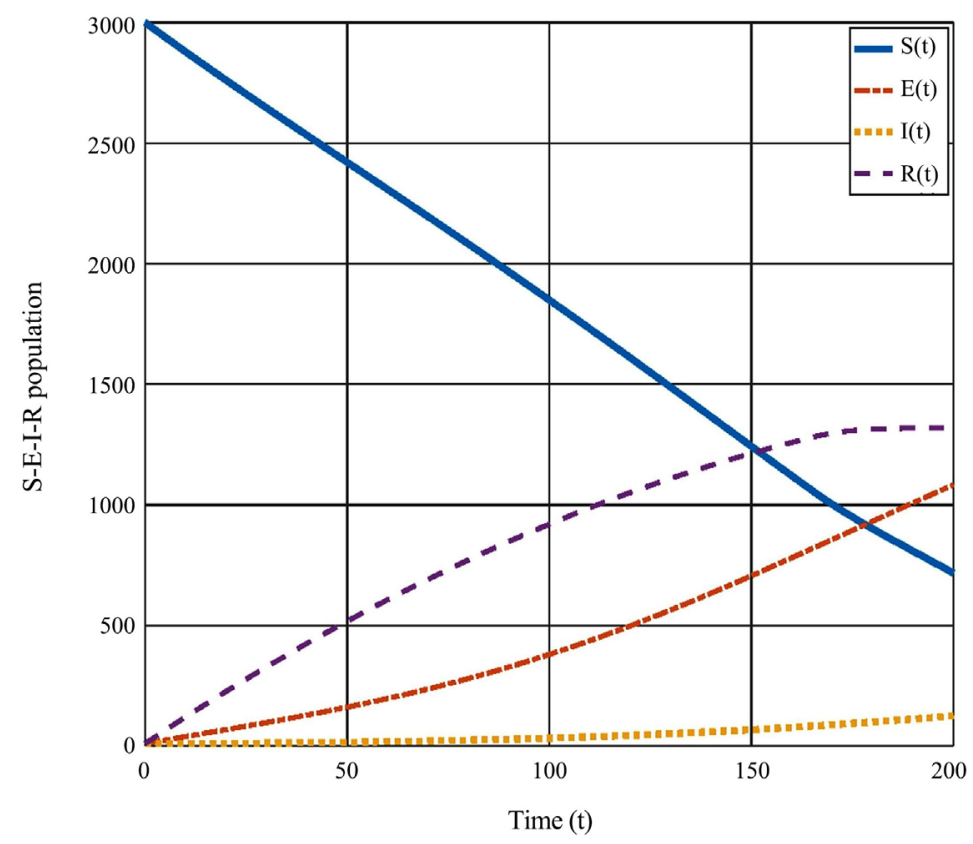

Figure 12. SEIR-Compartment with Optimal Control at $\mathrm{u}[0,0.75]$.

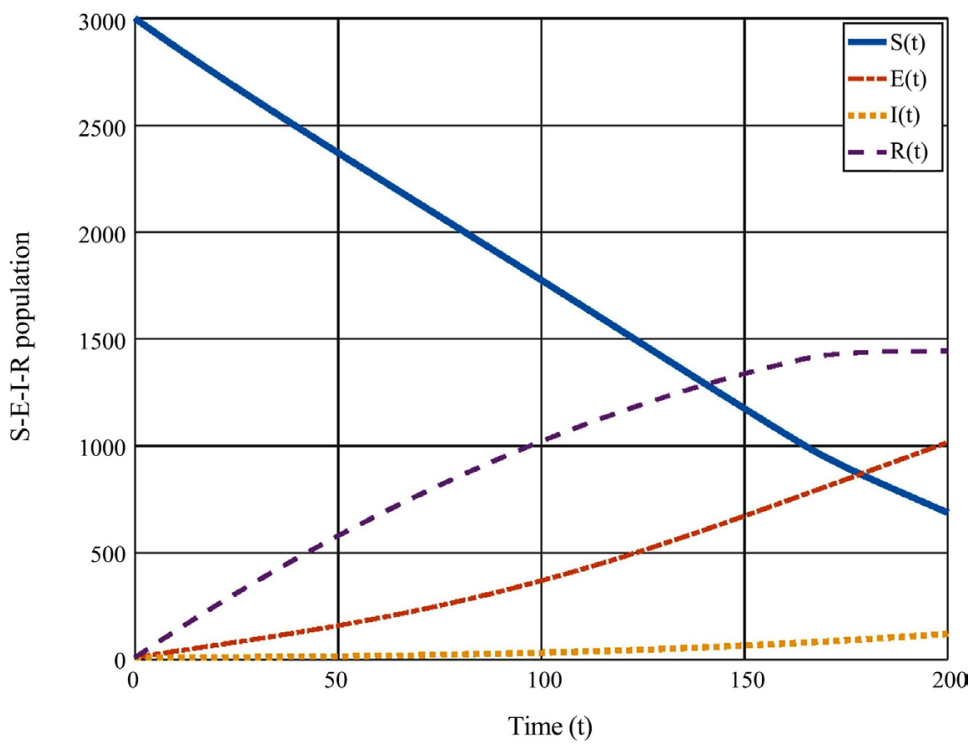

Figure 13. SEIR-Compartment with Optimal Control at u [0, 0.85].

\section{Conclusions}

The work reported herein demonstrates the application of vaccination as an optimal control strategy for LSD cattle disease in Ethiopia in addition to the optimization of the cost of the protective strategy adopted. To implement this, we used the Pontryagins maximum principle to characterize the controls and derive the optimality system. For the numerical simulation, we adopted an algorithm based on the Runge-Kutta forward-backward sweep method. The state equations were solved forward in time, while the adjoint equations were simultaneously solved backward in time. 
Both the controlled and uncontrolled scenarios were demonstrated graphically in order to identify the optimal vaccination schedule for the treatment and assessment of the impact of LSD on the cattle population. The results of our numerical experiments (Figures 8-13) clearly demonstrate that the applied optimality measures and intervention protocol have a very desirable effect in controlling and reducing the number of infected cattle population during the control period as long as they are properly managed. In addition, as the control period increases the number of cattle population saved from LSD gradually increased.

Based on our LSD model and parameters adopted for this study, the effects of the optimal vaccination strategy can be assessed for the vaccination rates in the range of $(0 \leq v \leq 0.85)$. It is observed that the control measures adopted herein achieves optimal operation at approximately $v=0.6$.

\section{Conflicts of Interest}

The authors declare no conflicts of interest regarding the publication of this paper.

\section{References}

[1] Abera, Z., Degefu, H., Gari, G. and Ayana, Z. (2015) Review on Epidemiology and Economic Importance of Lumpy Skin Disease. International Journal of Basic and Applied Virology, 4, 8-21.

[2] Birhanu, H. and Gezahign, A. (2015) Epidemiology, Economic Importance and Control Techniques of Lumpy Skin Disease: A Review. International Journal of Agricultural Research and Review, 3, 197-205.

[3] Regbe, H. (2012) Lumpy Skin Disease (LSD): Outbreak Investigation, Isolation and Molecular Detection of LSD in Selected Areas of Eastern Shewa, Ethiopia. Master Thesis, The School of Graduate Study of Addis Ababa University.

[4] Alaa, A., Hatem, M. and Khaled, A. (2008) Polymerase Chain Reaction for Rapid Diagnosis of a Recent Lumpy Skin Disease Virus Incursion to Egypt. Arab Journal of Biotechnology, 11, 293-302.

[5] Hawsar, Y.A., Heshu, S.R., Hiewa, O.D. and Hemn, H.O. (2016) Lumpy Skin Disease. Reproductive Immunology, 1, 25.

[6] Birhanu, H., Gezahign, A. and Nuru, S. (2015) Techniques of Lumpy Skin Disease. Animal. https://doi.org/10.11648/j.avs.20150302.15

[7] Davies, G. (1991) Lumpy Skin Disease, an African Capripox Virus Disease of Cattle. The Veterinary Journal, 147, 489-502. https://doi.org/10.1016/0007-1935(91)90019-J

[8] OIE (2010) OIE Manual of Diagnostic Tests and Vaccines for Terrestrial Animals. Lumpy Skin Disease, 14, 768-778.

[9] Tuppurainen, E.S.M. and Oura, C.A.L. (2012) Review: Lumpy Skin Disease: An Emerging Threat to Europe, the Middle East and Asia. Transboundary and Emerging Diseases, 59, 4048. https://doi.org/10.1111/j.1865-1682.2011.01242.x

[10] Chihota, C., Rennie, L.S., Kitching, R.P. and Mellor, P.S. (2001) Mechanical Transmission of Lumpy Skin Disease Virus by Aedes aegypti (Diptera: Culicidae). Epi- 
demiology \& Infection, 126, 317-321. https://doi.org/10.1017/S0950268801005179

[11] Gari, G., Waret-Szkuta, A., Grosbois, V., Jacquite, P. and Roger, F. (2010) Risk Factors Associated with Observed Clinical Lumpy Skin Disease in Ethiopia. Epidemiology \& Infection, 138, 1657-1666. https://doi.org/10.1017/S0950268810000506

[12] Lenhart, S., Bodine, E., Zhong, P. and Joshi, H. (2013) Illustrating Optimal Control Applications with Discrete and Continuous Features. Fields Institute Communications, 66, 209-238. https://doi.org/10.1007/978-1-4614-5389-5_9

[13] Lenhart, S. and Workman, J.T. (2007) Optimal Control Applied to Biological Models. Mathematical and Computational Biology Series, Chapman and Hall/CRC.

[14] Bubniakov, L. (2000) The Mathematics of Infectious Diseases. SIAM, 42, 599-653. 